\title{
Deployment of a Persistent Underwater Acoustic Sensor Network: The CommsNet17 Experience
}

\author{
Roberto Petroccia, ${ }^{*}$ Jan Śliwka, ${ }^{*}$ Alberto Grati, ${ }^{*}$ Vittorio Grandi, ${ }^{*}$ Piero Guerrini, ${ }^{*}$ \\ Andrea Munafò ${ }^{\dagger}$ Marin Stipanov, ${ }^{*}$ João Alves* and Robert Been* \\ * NATO STO Centre for Maritime Research and Experimentation \\ Viale S. Bartolomeo 400, 19126 La Spezia, Italy \\ email: \{roberto.petroccia; jan.sliwka; alberto.grati; vittorio.grandi; piero.guerrini; \\ marin.stipanov; joao.alves; robert.been\}@cmre.nato.int \\ $\dagger$ Marine Autonomous \& Robotic Systems (MARS), National Oceanography Centre \\ European Way, Southampton, SO14 3ZH, UK \\ email: andmun@noc.ac.uk
}

\begin{abstract}
This paper presents the experimental activities performed by the NATO STO Centre for Maritime Research and Experimentation (CMRE) during the CommsNet17 trial where a persistent Underwater Acoustic Sensor Network (UASN) was deployed. The CommsNet17 trial was held from the $27^{\text {th }}$ of November to the $6^{\text {th }}$ of December in the Gulf of La Spezia (IT), close to the CMRE premises, using the CMRE Littoral Ocean Observatory Network (LOON) as one of its key components. A network consisting of up to eleven nodes was deployed, including static and mobile assets. Various aspects related to persistent UASNs were addressed, including autonomous and distributed network discovery and node configuration, node localisation and navigation, self-adjustment of the network topology in support to the assigned tasks, underwater docking, wireless battery recharging and data offloading. The collected results show that the employed solutions were able to successfully complete all these tasks, thus demonstrating the effective deployment of a persistent, distributed and ad-hoc UASN.

Index Terms-Underwater Acoustic Communications, Acoustic Networks, Network Protocols, Persistent Underwater Networks, Underwater Docking, Localisation.
\end{abstract}

\section{INTRODUCTION}

The interest on Underwater Acoustic Sensor Networks (UASNs) and on underwater robotics has largely increased in the past decade. This is a consequence of the fact that such enabling capabilities can support a wide range of emerging applications, including monitoring of the environment and critical infrastructures, coastline protection, measurement of underwater seismic and volcanic events [1], [2], to state only a few. For many of these applications the continuous monitoring of ocean processes is a must [3], [4]. This requires the deployment of persistent networks, able to adapt in realtime and in an ad-hoc way to possible network changes, e.g. node failure, addition and/or removal. To ensure a sufficient

This work was supported in part by the NATO Allied Command Transformation (ACT) Future Solutions Branch under the Autonomous Security Network Programme and the Office of Naval Research Global under grant no. N62909-16-1-2095. coverage of the target area and a reliable monitoring capability, these networks can be composed by a heterogeneous set of assets (cabled and wireless, static and mobile) which can include Autonomous Underwater Vehicles (AUVs), Unmanned Surface Vehicles (USVs), gliders, buoys, drifting nodes, and bottom-mounted nodes in any combination.

Recently, multiple works have been proposed where the use of collaborative heterogeneous underwater networks is presented. Such networks are intended to achieve the desired level of adaptation in performing the assigned tasks [5]-[9], as required by the envisioned applications. In these works, various challenges concerning the communication and cooperation objectives have been addressed. Main focus has been on localisation and navigation strategies, including their integration with the communications and networking solutions. However, aspects related to self-configuration and reconfiguration of the deployed network have been marginally addressed. Other topics not largely tackled are the ones directly related with persistence, namely the possibility for the AUVs to adjust their mission when a low battery level is detected in order to recharge the batteries and offload the collected data. This feature would enable these vehicles to operate over long periods (i.e., multiple days/months).

This work describes the research conducted at CMRE addressing these challenges and the deployment of a persistent UASN performed during the CommsNet17 trial. This trial was organised by CMRE and held from the $27^{\text {th }}$ of November to the $6^{\text {th }}$ of December in the Gulf of La Spezia (IT), close to the CMRE premises. A network consisting of up to eleven nodes was deployed, including static and mobile assets. The CMRE Littoral Ocean Observatory Network (LOON) [10] was used as the network backbone. To handle the communications and networking tasks, the Cognitive Communications Architecture (CCA) developed by CMRE was employed. Various important aspects for the deployment of a fully autonomous and persistent UASN have been considered, spanning from network 


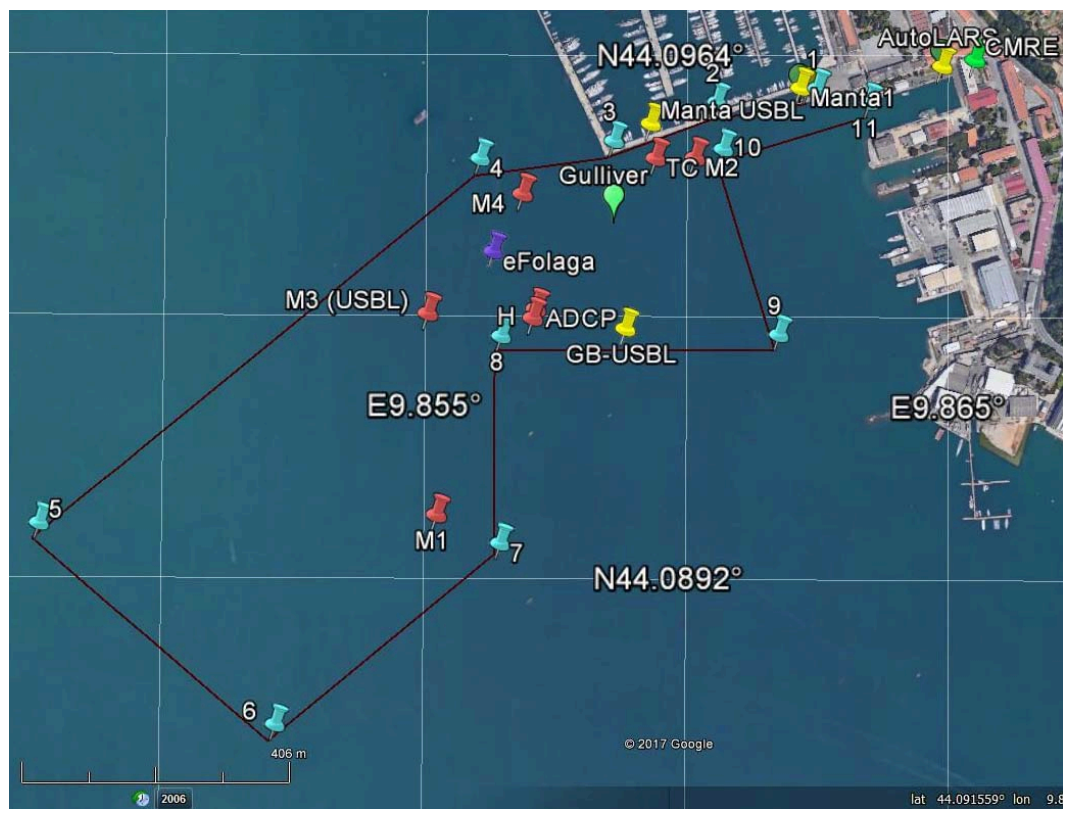

(a) Area of operation during CommsNet17.

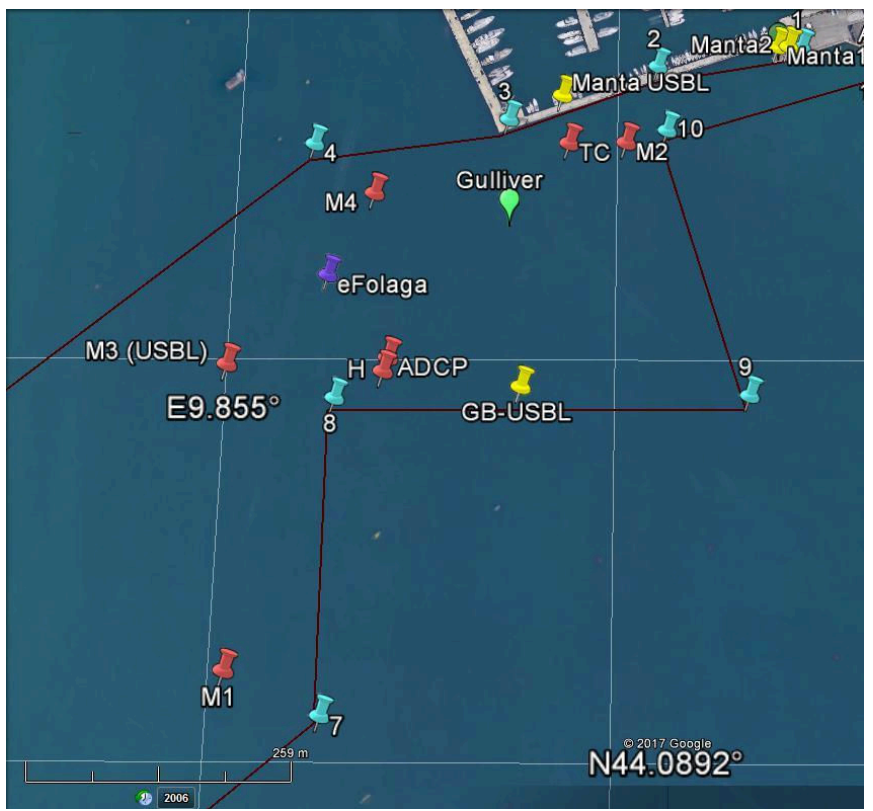

(b) Area of operation during CommsNet17 (Zoom into the LOON area).

Fig. 1: CommsNet17: area of operation and nodes deployment.

discovery and configuration to mobile assets navigation and underwater docking.

The collected results show that all these aspects and related objectives have been successfully demonstrated, under various configurations and scenarios. The deployed network was able to discover and configure all the eleven nodes in approximately two minutes, connecting nodes that were up to 4 hops apart. All the network was cooperating to support the localisation and navigation of mobile nodes, including automatic docking.

The rest of the paper is organised as follows. Section II details the CommsNet17 sea trial, the scientific objectives, the area of operations and the various assets involved. The employed algorithms and protocols are described in Section III along with the collected results. Finally, Section IV concludes the paper.

\section{COMmSNet17 SEA TRIAL}

This Section describes the CommsNet17 scientific objectives, including details about the area of operations and the deployed assets.

\section{A. Scientific objectives}

Various scientific objectives were defined by CMRE for the CommsNet17 trial. The ones related to the deployment of a persistent UASN are listed in what follows:

- validate the use of a network bootstrapping protocol to discover and self-organise the network under different configurations;

- evaluate the use of communications and networking solutions in support to the localisation and navigation of mobile vehicles;
- evaluate the use of autonomous behaviours to change the position of mobile nodes in order to optimise the execution of the localisation and navigation tasks;

- demonstrate the possibility to extend the duration of an AUV mission beyond the maximum battery endurance.

\section{B. Area of operations}

The area of operations for CommsNet17 is depicted in Figure 1a. The positions of the markers (light blue) delimiting the area of operations is reported in Table I. Figure $1 \mathrm{~b}$ zooms into the LOON area, including the LOON fixed nodes which are placed on the sea bottom. The position of the acoustic modems and environmental sensors is reported in Table II.

TABLE I: Position of the markers displayed in the area of operations.

\begin{tabular}{ccc} 
Marker & Latitude & Longitude \\
\hline 1 & $44^{\circ} 05.745^{\prime} \mathrm{N}$ & $009^{\circ} 51.737^{\prime} \mathrm{E}$ \\
2 & $44^{\circ} 05.731^{\prime} \mathrm{N}$ & $009^{\circ} 51.627^{\prime} \mathrm{E}$ \\
3 & $44^{\circ} 05.699^{\prime} \mathrm{N}$ & $009^{\circ} 51.506^{\prime} \mathrm{E}$ \\
4 & $44^{\circ} 05.684^{\prime} \mathrm{N}$ & $009^{\circ} 51.355^{\prime} \mathrm{E}$ \\
5 & $44^{\circ} 05.384^{\prime} \mathrm{N}$ & $009^{\circ} 50.853^{\prime} \mathrm{E}$ \\
6 & $44^{\circ} 05.218^{\prime} \mathrm{N}$ & $009^{\circ} 51.124^{\prime} \mathrm{E}$ \\
7 & $44^{\circ} 05.372^{\prime} \mathrm{N}$ & $009^{\circ} 51.381^{\prime} \mathrm{E}$ \\
8 & $44^{\circ} 05.540^{\prime} \mathrm{N}$ & $009^{\circ} 51.380^{\prime} \mathrm{E}$ \\
9 & $44^{\circ} 05.541^{\prime} \mathrm{N}$ & $009^{\circ} 51.701^{\prime} \mathrm{E}$ \\
10 & $44^{\circ} 05.696^{\prime} \mathrm{N}$ & $009^{\circ} 51.634^{\prime} \mathrm{E}$ \\
11 & $44^{\circ} 05.734^{\prime} \mathrm{N}$ & $009^{\circ} 51.807^{\prime} \mathrm{E}$ \\
\hline
\end{tabular}

\section{Main Equipment and Assets}

This Section lists the devices deployed during CommsNet17 for experimentation and scientific data collection. The key 
asset used during the trial was the LOON testbed. The LOON is a permanent testbed developed by CMRE which was designed with the objective to have a modular system enabling to plug additional equipment of interest. The LOON consists of four bottom-mounted tripods (M1, M2, M3 and M4), with distances between nodes ranging from $\sim 250$ to $\sim 700 \mathrm{~m}$. The tripods are fitted with different acoustic modems (Woods Hole Micro-Modem [11] and Evologics S2C medium frequency (18/34) modem [12]) and support arbitrary waveform transmission/recording. Each of these tripods is cabled to a shore control station that provides data connection and power supply. The LOON shore station (LOON container) runs all the software required to perform the experiments and control the nodes. The LOON includes also environmental sensors, i.e. a thermistor chain, sound velocity sensors, an Acoustic Doppler Current Profiler (ADCP) with waves measurement, and a meteorological station. This meteorological station is installed on top of the LOON container and it is able to measure wind speed and direction, temperature, atmospheric pressure and humidity. The availability of all these measurements is important to correlate the performance of the protocols with physical phenomena that may impact the sound propagation conditions.

TABLE II: Positions of the fixed assets (acoustic modems and environmental sensors) deployed in the LOON area.

\begin{tabular}{|c|c|c|}
\hline Marker & Latitude & Longitude \\
\hline M1 & $44^{\circ} 05.390^{\prime} \mathrm{N}$ & $009^{\circ} 51.309^{\prime} \mathrm{E}$ \\
\hline M2 & $44^{\circ} 05.686^{\prime} \mathrm{N}$ & $009^{\circ} 51.603^{\prime} \mathrm{E}$ \\
\hline M3 (USBL) & $44^{\circ} 05.556^{\prime} \mathrm{N}$ & $009^{\circ} 51.297^{\prime} \mathrm{E}$ \\
\hline M4 & $44^{\circ} 05.655^{\prime} \mathrm{N}$ & $009^{\circ} 51.405^{\prime} \mathrm{E}$ \\
\hline $\mathrm{ADCP}$ & $44^{\circ} 05.560^{\prime} \mathrm{N}$ & $009^{\circ} 50.421^{\prime} \mathrm{E}$ \\
\hline Thermistor Chain (TC) & $44^{\circ} 05.685^{\prime} \mathrm{N}$ & $009^{\circ} 51.558^{\prime} \mathrm{E}$ \\
\hline
\end{tabular}

To enlarge the size of the LOON acoustic network and to extend its operational area, additional assets were deployed. Table III briefly describes the seven additional underwater nodes and the Automatic Launch And Recovery System (AutoLARS docking station) used during CommsNet17.

All the nodes were equipped with Evologics S2C 18/34 acoustic modems. The node M3, the gateway buoy and one Manta node were equipped with USBL-capable versions of the same modem. Regular modems were used for the other nodes. In what follows additional details are provided about the deployment of the nodes and the positioning of the acoustic modems:

- the four LOON nodes are installed on the sea bottom at depth of about $10 \mathrm{~m}$. The acoustic modems are installed at $\sim 1.5 \mathrm{~m}$ from the sea bottom facing upwards;

- the gateway buoy is connected to a USBL modem deployed at a depth of $\sim 7.5 \mathrm{~m}$ facing upwards. The modem is cabled to the surface expression of the buoy (where the GPS and radio antenna are installed) with a weight to stretch the cable. The modem is therefore hanging and moving around the surface float;
TABLE III: Additional assets.

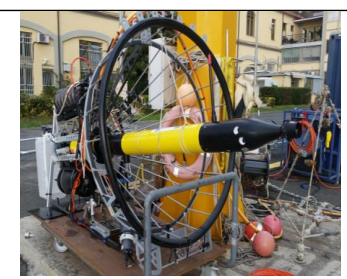

AutoLARS (x1): catcher structure with modified sensors suite, capable of detecting the presence and the fine alignment of the approaching AUV; Modified control software capable of automatic AUV locking/unlocking and battery charger engagement/disengagement;

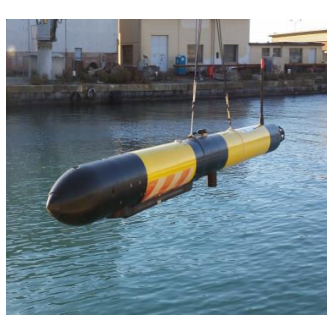

eFolaga (x1): small AUV with hovering capability, equipped with Evologics 18 $34 \mathrm{kHz}$ acoustic modem. It includes embedded boards to run the control, communications and localisation software. Its small size is highly beneficial to simplify deployment and recovery. It has a modified mission control software with automatic behaviour switching based on battery residual charge estimate. It is equipped with a docking algorithm with depth compensation, implemented in standard software behaviour;

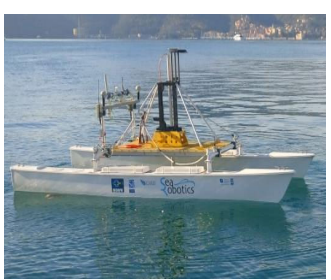

Gulliver (x1): self-propelled autonomous surface vehicle which was used as a relocatable gateway buoy or as a surface target to validate the localisation algorithms. It includes radio connectivity $(5 \mathrm{GHz})$, embedded boards to locally run the required software, RTK GPS, and an Evologics $18-$ $34 \mathrm{kHz}$ acoustic modem;

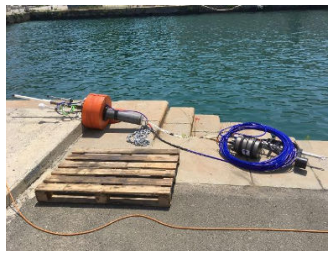

Portable drifting gateway buoy (x1): this includes dual radio connectivity (WiFi $2.4 \mathrm{GHz}$ and Freewave $900 \mathrm{MHz}$ ), an embedded board to locally run the required software, an Evologics $18-34 \mathrm{kHz}$ USBL acoustic modem. It can be easily deployed and recovered by two people from the RHIB. A light mooring was used during the trial keeping the buoy in position;

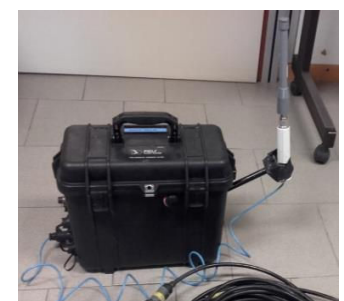

Manta portable node ( $x 4)$ : this includes radio connectivity (Wi-Fi $2.4 \mathrm{GHz}$ ), an embedded board to locally run the required software, an Evologics $18-34 \mathrm{kHz}$ acoustic modem. It can be easily relocated according to the experiments need. One of the four portable nodes is equipped with a USBLcapable version of the same modem.

- the USV is equipped with a modem installed on a rigid structure at a depth of $\sim 1.5 \mathrm{~m}$ facing downwards;

- three Manta nodes are connected to regular acoustic modems deployed from the pier at a depth of $\sim 2 \mathrm{~m}$ facing downwards;

- one Manta node is connected to a USBL installed on a small tripod and deployed from the pier. The use of the tripod avoids any movement of the USBL modem. The tripod is deployed at a depth of $\sim 10 \mathrm{~m}$ with the modem installed at $\sim 1 \mathrm{~m}$ from the sea bottom facing upwards;

- the eFolaga AUV has the modem installed on the hull of 
the vehicle facing downwards.

\section{PROTOCOL SOLUTIONS AND RESULTS}

This Section reports about the various tasks (listed in Section II-A) performed during CommsNet17 to support a fully autonomous and persistent UASN. A description of each task is provided, describing also the employed solutions and the collected experimental results. The combination of all these tasks enables the actual deployment and demonstration of a persistent, distributed and ad-hoc underwater system. To protect the network against the injection of fake messages that could compromise the correct execution of the various services, all acoustic messages were protected by the use of the cryptographic primitives supported by the CCA. These primitives combine the Advanced Encryption Standard (AES) [13] with the Galois Counter Mode (GCM) approach [14].

\section{A. Network discovery and configuration}

The ability to discover and self-configure the network nodes, along with the support for node addition and removal, are of paramount importance for the deployment of a persistent network. Additionally, reliability and robustness must be ensured in the presence of unreliable channels.

DIVE [15], standing for Distributed ID assignment and topology discoVEry, is a protocol developed by CMRE that has been used to tackle these challenges. It is fully distributed and self-adaptive and it does not assume the existence of any specific communications protocol. Similarly, it does not assume any knowledge about the deployed network, supporting the presence of both static and mobile devices. While discovering the network and assigning the node IDs, additional information is shared to support cooperative behaviours. Although DIVE has been proposed to self assign node IDs in the network, it can be also used as a leader election/consensus protocol to take other types of decisions, e.g. to define a hierarchical organisation of the network or distributed tasks allocation.

DIVE is designed to work in the presence of unreliable channels, such as the underwater acoustic one. Table IV reports the packet delivery ratio (PDR) monitored for the static nodes in the morning of the $5^{t h}$ of December, while performing DIVE experiments. It shows the presence of unreliable and asymmetric acoustic links. The computation of these values is based only on errors due to the acoustic channel with no packet collisions.

DIVE exploits link quality information to increase its reliability and robustness against message losses. During CommsNet17, DIVE performance were evaluated under a variety of network configurations, including node mobility along with node addition and removal, and exploring different network sizes. Two main scenarios were considered: one where all the nodes are deployed at the same time; another one where a first cluster composed by five nodes (LOON and a Manta node deployed from the LOON pier) is deployed and configured while additional nodes (from two to five) are added at a second stage. In the former case all the nodes need to self-discover and organise themselves, whereas in the latter case, the second cluster need to interact and coordinate with the first one in order to reach a common final configuration.

TABLE IV: PDR as monitored in the morning of the $5^{\text {th }}$ of December while performing DIVE experiments. M1-M4 are the LOON nodes, GB is the gateway buoy, MT refers to Manta nodes.

\begin{tabular}{|c|c|c|c|c|c|c|c|c|c|}
\hline & M1 & M2 & M3 & M4 & GB & MT-USBL & MT1 & MT2 & MT3 \\
\hline M1 & - & 0 & 0.12 & 0.28 & 0.40 & 0.18 & 0.22 & 0.22 & 0.16 \\
\hline M2 & 0 & - & 0 & 0 & 0.42 & 0 & 0.32 & 0.45 & 0.22 \\
\hline M3 & 0.53 & 0.03 & - & 0.5 & 0.53 & 0.28 & 0.28 & 0.43 & 0.37 \\
\hline M4 & 0.19 & 0 & 0.61 & - & 0.74 & 0.48 & 0.29 & 0.29 & 0.26 \\
\hline GB & 0.78 & 0.42 & 0.78 & 0.82 & - & 0.28 & 0.35 & 0.14 & 0.32 \\
\hline $\begin{array}{l}\text { MT- } \\
\text { USBL }\end{array}$ & 0.13 & 0.03 & 0.38 & 0.55 & 0.38 & - & 0.35 & 0.31 & 0.35 \\
\hline MT1 & 0 & 0.55 & 0.55 & 0.66 & 0.32 & 0.74 & - & 0.81 & 0.81 \\
\hline MT2 & 0.27 & 0.6 & 0.5 & 0.37 & 0.3 & 0.6 & 0.8 & - & 0.93 \\
\hline MT3 & 0.16 & 0.39 & 0.35 & 0.39 & 0.13 & 0.48 & 0.9 & 0.9 & - \\
\hline $\mathrm{R}$ & & 0.25 & DR & .50 & $5>=$ & 0.75 & & $=\mathrm{F}$ & $\varepsilon=1$ \\
\hline
\end{tabular}

Throughout all the experiments the DIVE protocol was $100 \%$ successful in discovering the network and converging to full network ID assignments.

Figure 2 shows the average number of seconds (Duration) required by DIVE to complete the node IDs assignment and network discovery process. Two curves are plotted, one where all the nodes are deployed together (ALL NODES), and a second one (CLUSTERS) for the cluster case. For the latter case, we only consider the time it takes to reach an agreement after the deployment of the second cluster, ignoring the time to create the first cluster.

Figure 3 shows the same curves for the average number of packets transmitted by each node.

As expected, when increasing the size of the network, the time to complete the DIVE protocol and the number of transmitted packets also increase. However, DIVE is designed in a way that enables each node to merge the received information before forwarding it to the others. This strategy enables to reduce the number of accesses to the channel and the traffic load when nodes are added to the network, thus being able to efficiently scale to larger scenarios.

\section{B. Node localisation and navigation}

The task of underwater node localisation and navigation has been addressed by employing the Network-based localisation (Net-LBL) solution [9], [16], [17]. This solution has been designed to support the localisation and navigation of underwater vehicles which are part of an underwater acoustic network. It can be employed for AUVs with poor dead reckoning capability, such as the eFolaga, or to improve the persistence of AUVs with good dead reckoning. The considered eFolaga does not have any navigation sensors, it is just provided with 


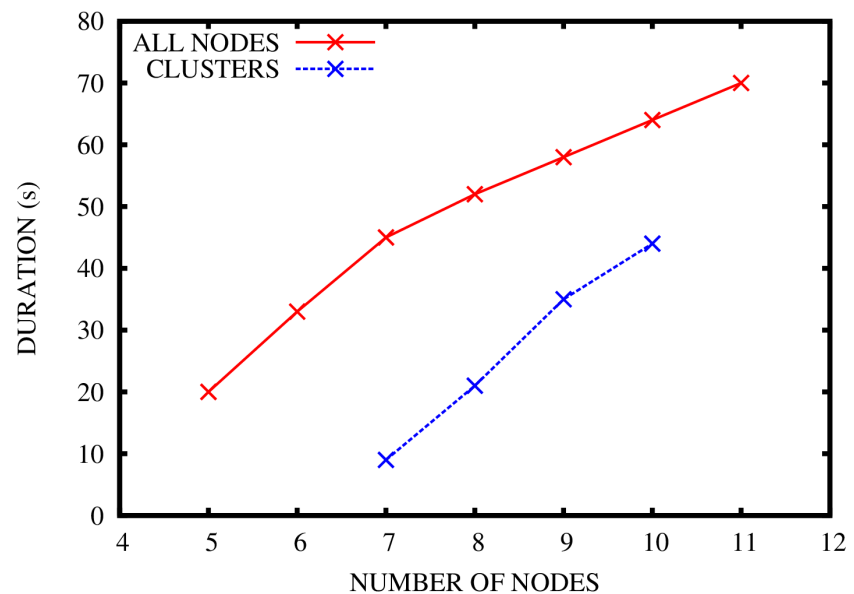

Fig. 2: Average number of seconds required by DIVE to complete the node IDs assignment and network discovery process for the two considered scenarios: All nodes and clusters.

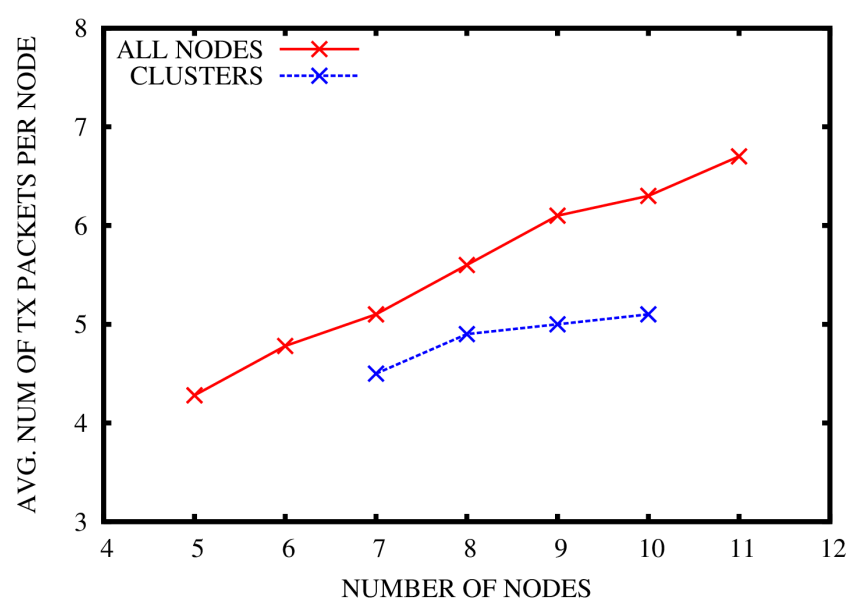

Fig. 3: Average number of packets transmitted by each node when using DIVE.

a compass (heading) and a thrust command (as a proxy of speed). Without any navigation aids it is very difficult for this vehicle to follow a given trajectory.

Net-LBL is based on the fact that all the nodes of the network can work as beacons, transmitting and receiving the intended data. The navigation filter is implemented using an analysis based on interval methods [18]. Two values are produced by the Net-LBL algorithm: the estimated position and the associated uncertainty, thus producing an upper bound on the localisation error.

Localisation information is added to the existing network messages so that every node of the network is able to correct its own position estimate, effectively performing concurrent communication and positioning. The Net-LBL system relies on range and bearing measurements (when available) in the network and in the exchange of node positions (for mobile assets) over time. The range and bearing data and the different proprioceptive measurements of the vehicle are then fed to the Net-LBL navigation algorithm which computes the position. Node position is computed in real-time and shared with the rest of the network to keep track of the changes in the topology due to the mobility of the nodes.

During CommsNet17, the Net-LBL system has been integrated with the CCA that provided the support for data exchange and range measurements in the network. Additionally, the CCA was reporting to the Net-LBL all the notifications coming from the acoustic modem, including bearing measurements, when available.

These two software modules have been integrated with the mission control software running on all the nodes which is based on MOOS [19]. Different message types were transmitted in support to localisation (ranging requests and replies, USBL measurements with respect to a target node, and estimated position) with different quality of service requirements. Additionally, status and report information from all the nodes were transmitted as well, as it would be in a real operational scenario. Various scenarios and configurations have been considered using the USV and the AUV as the target nodes requiring to localise themselves.

In what follows, preliminary results for the different components of the Net-LBL localisation algorithm are presented. A first assessment about the CCA ranging algorithm is reported, followed by the estimation of the localisation error and the trajectory reconstruction. The USV was used as the target node since it is equipped with a RTK GPS, providing a high quality ground truth. The trajectory followed by the USV is depicted in Figure 4.

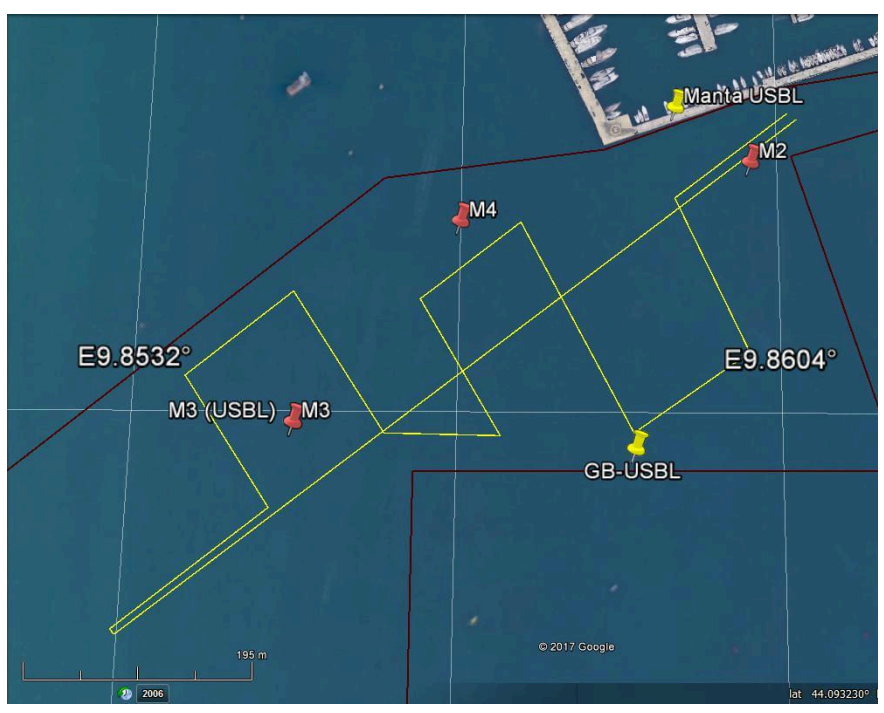

Fig. 4: Lawn mower and line trajectory. The mobile node starts the trajectory at the top right part of figure. It first follows the line trajectory and then returns following the lawn mower pattern. 
1) Cooperative ranging estimation: The CCA cooperative ranging estimation protocol [9], [20] was used to collect range measurements to be provided to the localisation service. This protocol is based on two-way time-of-flight measurements with multi-cast requests: single request and multiple replies. This enables the collection of multiple replies within a short time window. Replying nodes coordinate to avoid collisions at the requesting node. Additionally, the information about requests and replies can be piggybacked to regular data messages. The CCA algorithm does not compute the actual range measurement but only the propagation delay. This delay is then passed to the localisation algorithm and translated into the distance estimation using reference values for the sound velocity, which were collected by the environmental sensors of the LOON.

Figure 5 shows the average number of seconds it takes to receive ranging replies from multiple beacons in a network of 10 nodes with the USV following the trajectory depicted in Figure 4. Most of the time the USV was able to correctly

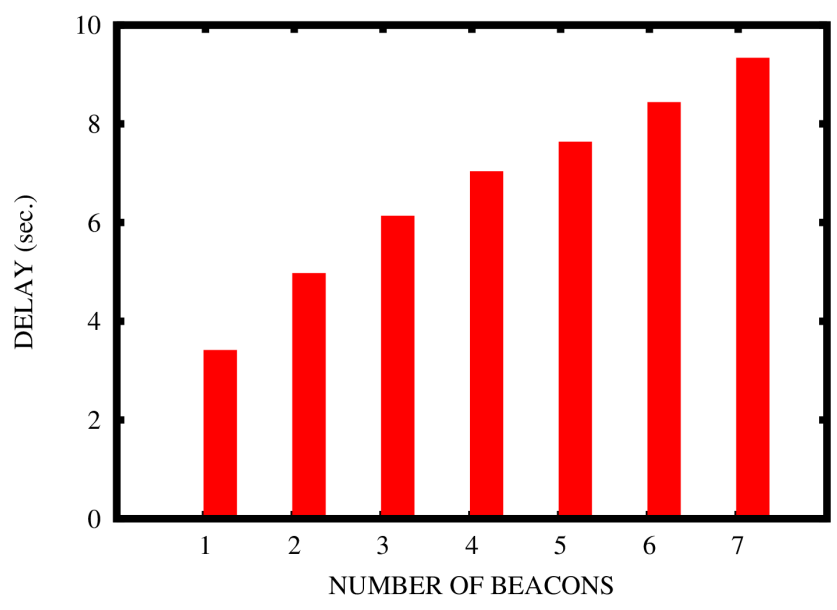

Fig. 5: Average delay to collect multiple range updates.

receive up to $3-4$ replies while navigating in the area, reaching a maximum number of 7 replies obtained in less than $10 \mathrm{~s}$. Shortening the delay between the transmission of a request and the reception of the replies enables to reduce possible errors introduced by the uncertainties in the movement of the involved nodes.

Figure 6 reports the error in the range measurements computed by the CCA algorithm when the link between M1 and M3 is considered: M1 sending the requests and M3 sending the replies. These two nodes are installed in fixed locations and there is a good knowledge of the distance between them. The result shows ranging errors always below $\pm 0.2 \mathrm{~m}$. Some of the remaining error can be due to various factors, including small fluctuation on the sound speed velocity and some bending in the propagation of the acoustic signal. When considering the ranging to a moving node, the error in the estimated value increases. This is mostly due to the vehicle motion. Some time is in fact required by the algorithm to collect the range measurements, as reported in Figure 5. During this time the

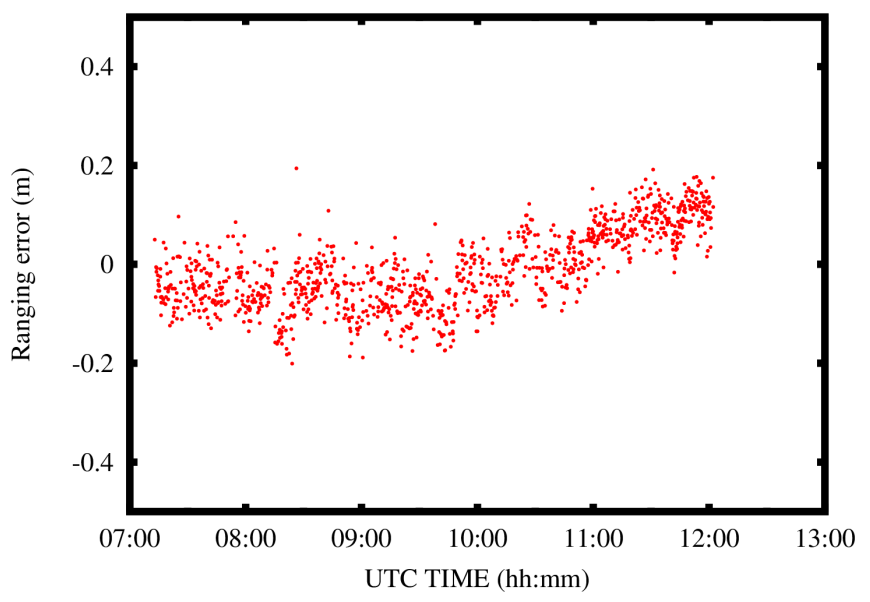

Fig. 6: Ranging error between M1 and M3.

vehicle could have moved, thus changing the actual distance between nodes. During CommsNet17 the USV was moving at a speed of $1-1.5 \mathrm{~m} / \mathrm{s}$ and an average error of few meters was reported. This error could be however reduced by adding more information in the ranging packets (i.e. transmission/reception timestamps ${ }^{1}$ ).

2) Localisation results: Figure 7 shows the estimated trajectory as produced by the Net-LBL solution running in real-time on board the USV. Only range measurements are considered, which are combined with nodes positions and on board sensor readings for USV speed and heading. This trajectory (also displayed in Figure 4) is compared with the one obtained using the on board RTK GPS. The speed of the USV is about $1-1.5 \mathrm{~m} / \mathrm{s}$.

The reconstructed trajectory is quite accurate with an average error on the computed position (black curve) of few meters, as reported in Figure 8. The Net-LBL uncertainty on the produced position is also reported in the figure (red curve). This uncertainty is produced by the interval methods based on the combination of the received measurements. Various factors can contribute to the uncertainty, including errors in the range measurements and less accurate nodes positions when a nondifferential GPS is used ${ }^{2}$. Non-differential GPS was installed on the Manta portable nodes and on the gateway buoy. The values for the uncertainty are however limited. This means that the positions produced by the algorithm are reliable.

The usage of this kind of cooperative approach, combining localisation with regular data transmission, opens to new scenarios where moving beacons can be employed, thus extending the operational area of the vehicles.

\footnotetext{
${ }^{1}$ For the considered solution, only the delay between the reception of the ranging request and the transmission of the reply is included into the ranging reply message.

${ }^{2}$ Non-differential GPS has an uncertainty in the reported position in the order of $3-5 \mathrm{~m}$.
} 


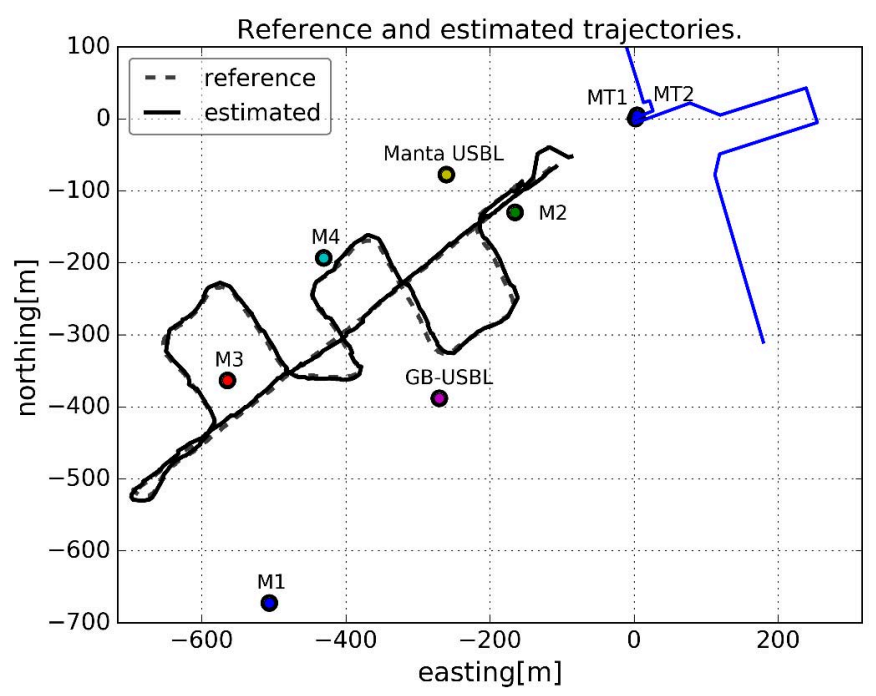

Fig. 7: USV trajectory reconstruction using the Net-LBL algorithm. The blue line represents the pier line.

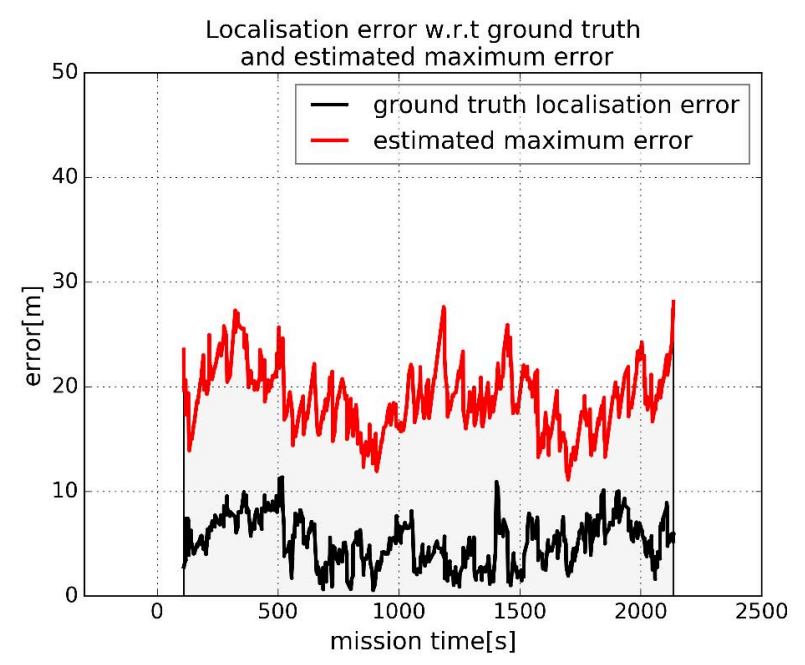

Fig. 8: Estimated localisation error using the Net-LBL algorithm.

\section{Network topology reconfiguration}

One of the task investigated during CommsNet17, for the deployment of a persistent UASN, has been the capability of adapting the network topology in support to the AUV navigation. More specifically, an autonomous behaviour was deployed on the USV to optimise its navigation with respect to the positions received from the AUV and the other beacons. The objective of this behaviour is to to provide a better network geometry for the Net-LBL execution.

Figure 9 shows the AUV (yellow triangle) moving in circle around M3 while the USV (red marker) is autonomously changing its positions over time. From the traces of the two mobile vehicles is clear how the USV is accompanying the AUV with its own movement. The decisions taken by USV are all computed in real-time on board the vehicle, based on the acoustic information received by the AUV and by the other

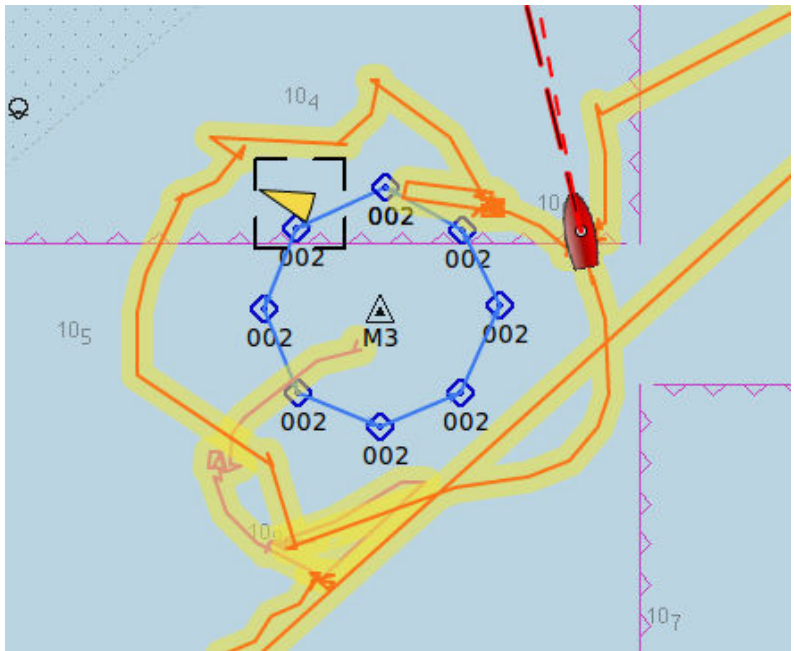

Fig. 9: USV (red marker) running an autonomous behaviour to act as mobile beacon for the AUV (yellow triangle).

nodes operating in the area [21].

\section{Automatic docking}

In the previous Sections the problem of discovering and configuring a network in a distributed and ad-hoc way has been presented. Similarly, the use of networked-based approach in support to mobile nodes localisation and navigation has been discussed, including the possibility to use mobile beacons and autonomously adjust the network geometry to perform the required tasks. The last step towards the deployment of a persistent UASN is the capability to extend the duration of an AUV mission beyond the maximum battery endurance.

To accomplish this task CMRE has developed the AutoLARS $^{3}$ described in Section II-C that provides the support for AUV docking, wireless battery recharging and high-speed data transfer. Additionally, the commercial version of the Folaga AUV [22] has been modified to enable the interaction with the docking station. Both the software and hardware of the commercial vehicle have been enhanced to demonstrate the envisioned capabilities, i.e. automatic docking, underwater wireless battery recharging and underwater high-speed data transfer. These features have been structured into an automated mission control software, in order to enable the automatic switching between a normal AUV mission and a "survival mode" mission. When in survival mode, the above mentioned features take over until AUV state (battery charge and onboard data storage capacity) is restored to conditions that allow the normal mission to be resumed.

To enable the deployment of a fully autonomous system, the mission control software of the AUV has been integrated with the communications and networking feature of the CCA and the localisation service provided by the Net-LBL.

When in low battery state, the AUV was using the CCA and Net-LBL solutions to navigate towards the docking station

\footnotetext{
${ }^{3}$ The AutoLARS has been developed by CMRE as part of the Persistent Autonomous Reconfigurable Capability (PARC) program.
} 


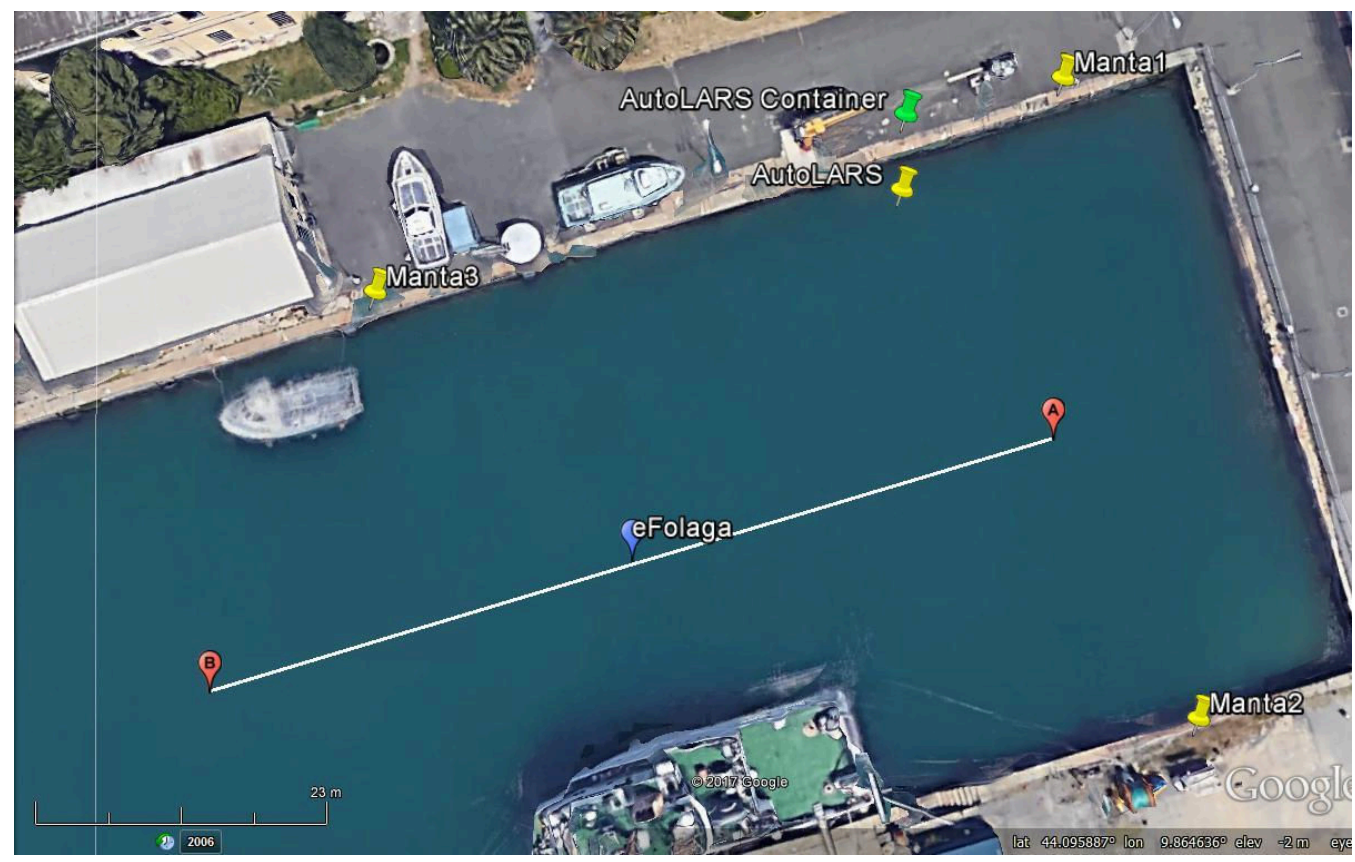

Fig. 10: Area of deployment of the AutoLARS (from the pier). It also displays the trajectory followed by the eFolaga to approach the docking station and the deployment of three portable nodes to support the navigation of the AUV within the small basin.

(deployed in the basin displayed in the top right part of Figure 1a), which is outside the LOON area. Figure 10 zooms into the area of deployment of the AutoLARS, displaying the line trajectory followed by the AUV to approach the AutoLARS and start the docking process. The AUV was moving from the point on the left of the figure (point $\mathbf{B}$ ) to the one on the right (point A). Three Manta nodes were deployed in the AutoLARS area to act as acoustic beacons while the AUV enters the basin, moves on the line trajectory to reach the target point, leaves the docking station to go back to the LOON area and continues the assigned mission.

Figure 11 shows the deployment of the AutoLARS before performing the docking. The docking process is composed of various steps. When the AUV reaches point $\mathbf{A}$, the automatic docking starts. Before engaging with the AutoLARS, the mission control software disables any CCA acoustic transmissions. This is to avoid possible interference with the docking process which is also based on the exchange of acoustic messages ${ }^{4}$. The AUV then starts autonomously interacting with the docking station to adjust its heading and speed in order to enter AutoLARS catcher structure. Once docked, the underwater wireless battery recharging and underwater high-speed data transfer start. After charging the battery and offloading the data, the AUV can perform the undocking. It reactivates the CCA transmissions and switches to the normal mission mode, returning to the LOON area and using again the CCA and Net-LBL in support to the assigned mission.

Using the described configuration and integrated solutions all these tasks have been successfully performed.

Figure 12 displays the AUV after the docking while recharg-

\footnotetext{
${ }^{4} \mathrm{~A}$ future step is to install and use the CCA on the AutoLARS.
}

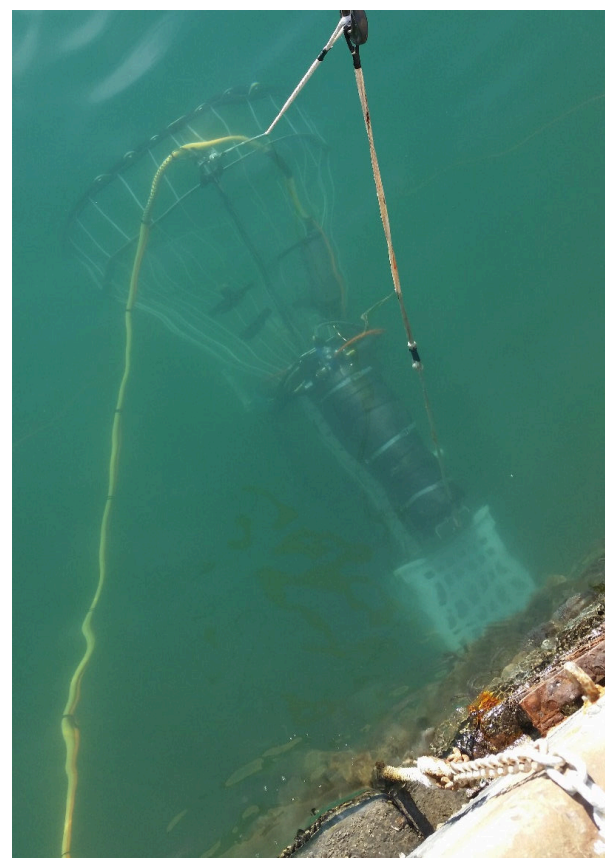

Fig. 11: Deployment of the AutoLARS in water, before performing the docking.

ing the battery and offloading the data. This process was correctly repeated multiple times to validate and demonstrate the whole integrated system and its single components.

\section{CONCLusions}

This paper presented the CommsNet17 sea trial activities as a demonstration of the effective deployment of a persistent, 


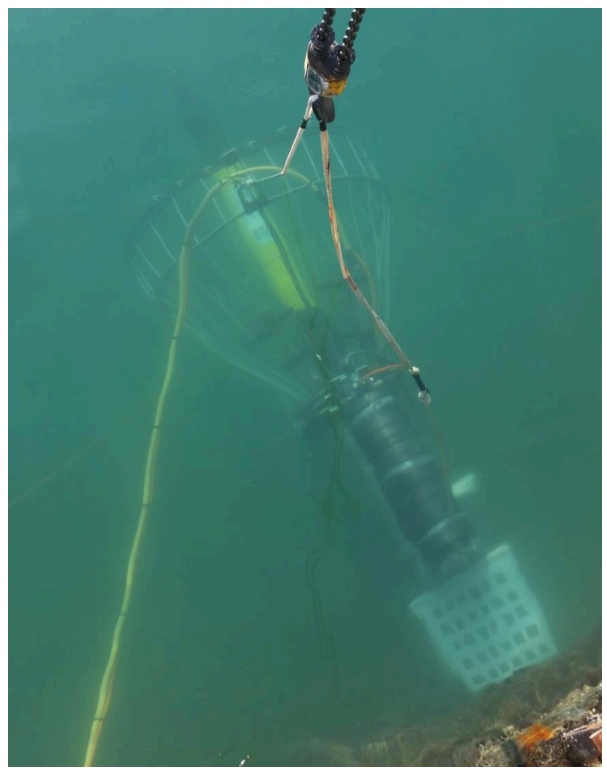

Fig. 12: Deployment of the AutoLARS in water, after the docking with the AUV inside the catcher structure.

distributed and ad-hoc UASN. A multi-hop network consisting of up to eleven nodes was deployed, including one AUV and one USV, exploring different configurations and scenarios. All the key objectives to support the deployment of such a persistent network were successfully achieved. The use of a network bootstrapping protocol to discover and self-organise the network under different configurations was validated. The combination of communications and networking to support the real-time localisation and navigation of mobile robots was demonstrated. The use of mobile beacons in support of AUV operations and the possibility to autonomously adjust the network topology, to facilitate the assigned tasks, were positively evaluated. Finally, the possibility to extend the duration of an AUV mission beyond the maximum battery endurance, including automatic docking, underwater wireless battery recharging and high-speed data transfer were effectively demonstrated.

The combination of all these aspects in a single integrated system (composed of heterogeneous assets) opens the way to new possible applications that require continuous operations, such as monitoring of long-term ocean processes, effective pipeline inspection and surveillance, to state only a few.

\section{ACKNOWLEDGEMENTS}

This work acknowledges the use of data that was made possible by the CommsNet17 sea trial, including as participants CMRE and CSSN. The authors would also like to acknowledge and thank everyone that contributed to the CommsNet17 sea trial.

\section{REFERENCES}

[1] N. Meratnia, P. J. M. Havinga, P. Casari, C. Petrioli, K. Grythe, T. Husoy, and M. Zorzi, "CLAM-collaborative embedded networks for submarine surveillance: An overview," in Proceedings of IEEE/OES OCEANS 2011, June 6-9 2011.
[2] J. Heidemann, M. Stojanovic, and M. Zorzi, "Underwater sensor networks: Applications, advances, and challenges," Royal Society, vol. 370, no. 1958 , pp. $158-175$, May 2012.

[3] A. Y. Martin, "Unmanned maritime vehicles: Technology evolution and implications," Journal of Maritime Technology Society, vol. 47, no. 5, pp. 72-83, September/October 2013.

[4] S. L. Wood and C. E. Mierzwa, "State of technology in autonomous underwater gliders," Journal of Maritime Technology Society, vol. 47, no. 5, pp. 84-96, September/October 2013.

[5] N. A. Cruz, B. M. Ferreira, O. Kebkal, A. C. Matos, C. Petrioli, R. Petroccia, and D. Spaccini, "Investigation of underwater acoustic networking enabling the cooperative operation of multiple heterogeneous vehicles," Marine Technology Society Journal, vol. 47, pp. 43-58, March/April 2013.

[6] A. Munafò, T. Furfaro, G. Ferri, and J. Alves, "Supporting AUV localisation through next generation underwater acoustic networks: Results from the field," in Intelligent Robots and Systems (IROS), 2016 IEEE/RSJ International Conference on. Daejeon, Korea: IEEE, October 9-14 2016, pp. 1328-1333.

[7] B. Allotta, F. Fanelli, N. Monni, C. Petrioli, L. Picari, A. Ridolfi, and D. Spaccini, "Enabling cooperation and networking in heterogeneous underwater networks composed of multi-vendor vehicles and modems," in Proceedings of MTS/IEEE OCEANS 2017, Aberdeen, Scotland, June 19-22 2007.

[8] A. Munafò and G. Ferri, "An acoustic network navigation system," Journal of Field Robotics, vol. 34, no. 7, pp. 1332-1351, March 2077.

[9] J. Sliwka, R. Petroccia, A. Munafò, and V. Djapic, "Experimenta evaluation of Net-LBL: An acoustic network-based navigation system," in Proceedings of MTS/IEEE OCEANS 2017, Aberdeen, Scotland, June 19-22 2017

[10] J. Alves, J. R. Potter, P. Guerrini, G. Zappa, and K. LePage, "The LOON in 2014: Test bed description," in Proceedings of the 2nd IEEE International Conference on Underwater Communications and Networking, ser. UComms14, Sestri Levante, Italy, September, 3-5 2014.

[11] L. Freitag, M. Grund, S. Singh, J. Partan, P. Koski, and K. Ball, "The WHOI Micro-Modem: An acoustic communications and navigation system for multiple platforms," https://acomms.whoi.edu/micro-modem/, 2005, Last time accessed: March 2018

[12] Evologics, "Evologics S2C acoustic modems," Last time accessed: March 2018. [Online]. Available: http://www.evologics.de/

[13] Wikipedia, Advanced Encryption Standard, Last time accessed: March 2018.

[14] M. J. Dworkin, "NIST SP 800-38D. Recommendation for Block Chiper Modes of Operation: Galois/Counter Mode (GCM) and GMAC," Technical report, Gaithersburg, MD, USA, 2007.

[15] R. Petroccia, "A distributed ID assignment and topology discovery protocol for underwater acoustic networks," in Proceedings of the 3rd IEEE International Conference on Underwater Communications and Networking, ser. UComms16, Lerici, Italy, August 30 - September 1 2016.

[16] J. Śliwka, A. Munafò, G. Ferri, and J. Alves, "Interval methods based AUV localisation in the context of an acoustic network with experimental results," in Proceedings of MTS/IEEE OCEANS 2014, St. John's, Canada, September 14-19 2014.

[17] A. Munafò, J. Śliwka, and R. Petroccia, "Localisation using undersea wireless networks," in Proceedings of MTS/IEEE OCEANS 2018, Kobe, Japan, May 28-31 2018.

[18] L. Jaulin, Applied interval analysis: with examples in parameter and state estimation, robust control and robotics. Springer Science \& Business Media, 2001, vol. 1.

[19] "MOOS: Mission oriented operating suite," Last time accessed: March 2015. [Online]. Available: http://www.moos-ivp.org

[20] J. Braga, R. Martins, C. Petrioli, R. Petroccia, and L. Picari, "Cooperation and networking in an underwater network composed by heterogeneous assets," in Proceedings of MTS/IEEE OCEANS 2016 , Monterey, CA, USA, September, 19-23 2016, pp. 1-9.

[21] A. Munafò, J. Śliwka, and J. Alves, "Dynamic placement of a constellation of surface buoys for enhanced underwater positioning," in Proceedings of MTS/IEEE OCEANS 2015, Genova, Italy, May 18-21 2015, pp. 1-6.

[22] Graaltech, "Folaga autonomous underwater vehicle," Last time accessed: March 2018. [Online]. Available: https://www.graaltech.com/folagafeatures 